\title{
Maintenance Heavy Equipment Management Through Contract Models or Company it Self
}

\author{
Andi Afiyuddin and Adithya Sudiarno \\ Department of Industrial and Systems Engineering, Institut Teknologi Sepuluh Nopember, Surabaya \\ E-mail: a.afiyuddin@gmail.com
}

\begin{abstract}
One important activity and cause high costs in the company is maintenance and in mining one of critical maintenance is heavy equipment. Issue of manpower, skill, expert and equipment population made company can't handle the maintenance it self. Agreement to the contractor made with several contract models. This maintenance management either by company it self or by different contract models have their strong and weakness point. This research will analyze which maintenance management is better using Fuzzy AHP as a multicriteria decision making tool. Responden will selected to define the criteria and sub-criteria. The result of this research are to select maintenance management that will use as standard in the company.
\end{abstract}

Keywords-Contract, Fuzzy AHP, Maintenance.

\section{BACKGROUND}

$\mathrm{P}$ T. Vale Indonesia is mining companies that produce nickel in Indonesia. PT. Vale Indonesia has a nickel refining facility in Sorowako, South Sulawesi. PT. Vale Indonesia covers 70,566 Ha in South Sulawesi, 22,699 Ha in Central Sulawesi and Southeast Sulawesi covering 24,752 Ha. PT Vale Indonesia mines nickel in the form of nickel ore and processing into nickel matte.

PT. Vale Indonesia in its operations both in the mining process or nickel refining process using a variety of equipment. The mining process uses heavy equipment such as: (1) Haul truck that serves to transport mined rocks to be brought to the processing area. (2) Dozer which functions to push or dig soil or rocks. (3) Wheel loader to move rocks or lift rocks into trucks. (4) Excavator to dig soil or rocks. (5) Graders to level the ground. Total heavy equipment at PT. Vale is 347 Units for the Caterpillar, Komatsu and Hitachi brands, with detail in Table 1.

This equipment operates 24 hours, and is operated by operators with 3 shifts. Heavy equipment is very critical, without this equipment the mining process will stop. Therefore, to ensure that the equipment operates optimally, a maintenance strategy is needed. A large number of units and variations, both from type, brand and age of the equipment, resulting in a maintenance process at PT. Vale becomes very complex. So, PT. Vale cooperates with the contractor for maintenance some equipment. This collaboration is in the form of a maintenance contract that is being processed by the procurement team.

Maintenance equipment performed by contractors has 3 contract schemes, which is:

\section{A. Marc Contract}

Where the full maintenance contract will be carried out by contractors for the service and spare part with lump sum payments per month. Currently the heaviest equipment units at PT. Vale carries out maintenance with this type of contract.

\section{B. SSA Contract}

Maintenance contract will be carried out jointly between the contractor and PT. Vale. The contractor will perform maintenance for services while PT. Vale will contribute to the spare parts needed by maintenance.

\section{On Call Contract}

Maintenance contract will be carried out jointly between the contractor and PT. Vale where contractor maintenance activities are categorized into several activities / work packages.

The choice of maintenance strategy requires a method for assessing from many of the criteria that arise from maintenance problems. One method for multi-criteria decision making is the Analytic Hierarchy Process. AHP method is often used in previous studies related to suppliers/contractors to determine supplier performance, determine supplier selection. Previous studies have used AHP to determine the type of contract awarded, previous research on grocery stores (S. M. Tazim Ahmed \& Chitra Karmaker, Journal of Supply Chain Management Systems). In this study using fuzzy AHP, which is a method of combining fuzzy and AHP which is better at solving problems. In contrast to this study, where previously the research was for contracts for providers of foodstuffs to be sold, whereas this study was for heavy equipment maintenance service contracts.

The objective of this research is: (1) Knowing the criteria for determining system maintenance. (2) Determine the maintenance system that should be the standard to use in the company.

\section{PROPOSED APPROACH}

\section{A. $A H P$}

The AHP developed by Professor Thomas Saaty in 1980 made it possible to arrange decisions hierarchically (to reduce their complexity) and show the relationship between goals (or criteria) and possible alternatives. Perhaps the greatest advantage of this method is that it allows the inclusion of intangible objects such as experience, subjective preferences and intuition, in a logical and structured manner. the greatest advantage of this method is that it allows the inclusion of intangible objects such as experience, subjective preferences and intuition, in a logical and structured manner. This research use AHP for 3 following steps. 
The $6^{\text {th }}$ International Seminar on Science and Technology (ISST) 2020

July $25^{\text {th }}$ 2020, Institut Teknologi Sepuluh Nopember, Surabaya, Indonesia

Table 1.

Total heavy equipment in PT. Vale

\begin{tabular}{ll}
\hline Manufacture & Unit \\
\hline Caterpillar & 248 \\
Hitachi & 27 \\
Komatsu & 72 \\
\hline \hline
\end{tabular}

Table 2.

Comparison scale

\begin{tabular}{ll}
\hline \hline Scale & Linguistic Variable \\
\hline 1 & Equally Important \\
3 & Weakly Important \\
5 & Strongly Important \\
7 & Very strongly Important \\
9 & Extremely Important \\
$2,4,6,8$ & Intermediate value between adjacent scales \\
\hline \hline
\end{tabular}

Table 3.

Fuzzy triangle scale

\begin{tabular}{|c|c|c|c|c|}
\hline $\begin{array}{l}\text { Linguistic } \\
\text { variable }\end{array}$ & Scale & TFN & Scale & TFN \\
\hline \multirow{4}{*}{$\begin{array}{l}\text { Equally } \\
\text { Important }\end{array}$} & & $(1,1,1)$ if & & $(1 / 1,1 / 1,1 / 1)$ \\
\hline & 1 & diagonal & $1 / 1$ & if diagonal \\
\hline & & $(1,1,3)$ & $1 / 1$ & $(1 / 1,1 / 1,1 / 3)$ \\
\hline & 2 & beside & 10 & beside \\
\hline Weakly & 3 & $(1,3,5)$ & $1 / 3$ & $(1 / 5,1 / 3,1 / 1)$ \\
\hline Important & 4 & $(2,4,6)$ & $1 / 4$ & $(1 / 6,1 / 4,1 / 2)$ \\
\hline Strongly & 5 & $(3,5,7)$ & $1 / 5$ & $(1 / 7,1 / 5,1 / 3)$ \\
\hline Important & 6 & $(4,6,8)$ & $1 / 6$ & $(1 / 8,1 / 6,1 / 4)$ \\
\hline Very strongly & 7 & $(5,7,9)$ & $1 / 7$ & $(1 / 9,1 / 7,1 / 5)$ \\
\hline Important & 8 & $(6,8,10)$ & $1 / 8$ & $(1 / 10,1 / 8,1 / 6)$ \\
\hline $\begin{array}{l}\text { Extremely } \\
\text { Important }\end{array}$ & 9 & $(7,9,11)$ & $1 / 9$ & $(1 / 11,1 / 9,1 / 7)$ \\
\hline
\end{tabular}

\section{1) Construct Hierarchy}

Creating hierarchical forms of various elements as principle objectives, goals that are influenced by these criteria or criteria that are influenced by sub-criteria and are nothing but different substitutes available for the problem (Figure 1).

\section{2) Pair-Wise Comparison (Determining Weights)}

The comparison matrix between factors is the $n \times n$ dimension box matrix. The matrix component on the diagonal of this matrix takes 1 value. When $\mathrm{i}=\mathrm{j}$, the component on the diagonal of the comparison matrix takes 1 value; because the related factor has compared with itself in this situation. Comparing factors is done according to their importance to each other and reciprocally. One-by-one and reciprocally comparing the importance scale of factors, using a comparison scale in Table 2.

\section{3) Consistency in Factor Comparisons was Calculated}

AHP suggests a process for measuring the consistency of this comparison. Finally, by obtaining a Consistency Ratio (CR), there has been an opportunity to test the consistency of priority vectors and also the consistency of pair comparisons between criteria. The essence of CR calculation is based on comparison of the number of criteria and coefficients, called the Main Value $(\chi)$ by the AHP. In principle, from doubling the comparison matrix $\mathrm{A}$ and priority vector $\mathrm{W}$, column vector $\mathrm{D}$ is obtained for calculation $\chi$

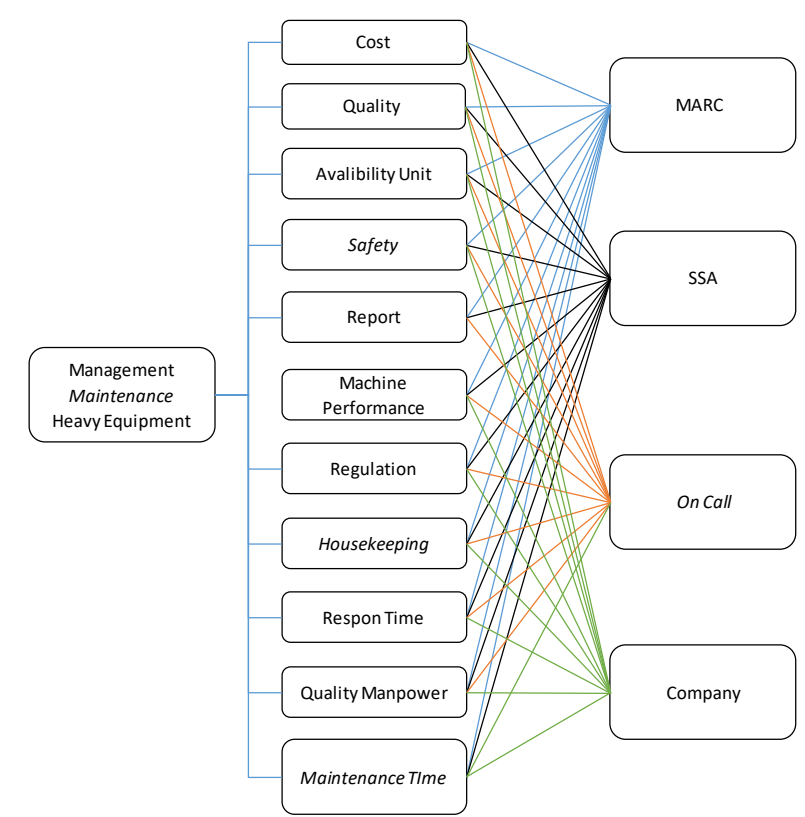

Figure 1. Hierarchical structure of maintenance system assessment.

$$
D=\left[\begin{array}{cccc}
a_{11} & a_{12} & \ldots & a_{1 n} \\
a_{21} & a_{22} & \ldots & a_{2 n} \\
\vdots & \vdots & & \vdots \\
a_{n 1} & a_{n 2} & \ldots & a_{n n}
\end{array}\right] x\left[\begin{array}{c}
w_{1} \\
w_{2} \\
\vdots \\
w_{n}
\end{array}\right]
$$

After $\chi$ is calculated, the Consistency Index (CI) can be calculated according to the following formula.

$$
C I=\frac{\lambda-n}{n-1}
$$

And finally calculate the value of the ratio consistency with the formula.

$$
C R=\frac{C I}{R I}
$$

Consistency is acceptable if the CR value $<0,100$.

\section{B. Fuzzy AHP}

AHP combined with fuzzy logic known as Fuzzy AHP is a popular method for dealing with uncertainty and helps decision makers in complex problems with various conflicting criteria (Kubler et al., 2016). The Fuzzy AHP model (FAHP) is based on fuzzy set theory, where the membership of a given element is determined by the membership function. The value of the fuzzy decision variable is explained by the membership function which is between zero and one.

Chang (1996) defines AHP intensity values into triangular fuzzy scales. The fuzzy triangle scale used by Chang can be seen in Table 3. Fuzzy AHP step in research as following:

\section{1) Calculate the Average Value of Fuzzy Geometric}

The initial step is to determine the geometric mean of Fuzzy numbers with the formula:

$$
\widetilde{r}_{l}=\left(\prod_{j=1}^{n} \tilde{C}_{i j}\right)^{1 / n}
$$

\section{2) Calculate the Value of Fuzzy Weights}

Calculate the value of Fuzzy weights with the formula:

$$
\begin{gathered}
\widetilde{w_{l}}=\widetilde{r_{l}} \otimes\left(\widetilde{r_{1}} \otimes \widetilde{r_{2}} \otimes \ldots \otimes \widetilde{r_{n}}\right)^{-1} \\
\widetilde{w_{l}}=\left(l w_{i}, m w_{i}, u w_{i}\right)
\end{gathered}
$$


The $6^{\text {th }}$ International Seminar on Science and Technology (ISST) 2020

July $25^{\text {th }} 2020$, Institut Teknologi Sepuluh Nopember, Surabaya, Indonesia

Table 4.

Matrix criteria of respondent 1

\begin{tabular}{|c|c|c|c|c|c|c|c|c|c|c|c|c|}
\hline Criteria & & $\mathrm{C} 1$ & $\mathrm{C} 2$ & $\mathrm{C} 3$ & $\mathrm{C} 4$ & $\mathrm{C} 5$ & C6 & C7 & C8 & C9 & $\mathrm{C} 10$ & C11 \\
\hline $\mathrm{C} 1$ & $\mathrm{R} 1$ & 1 & 0,33 & 0,33 & 0,17 & 5 & 0,33 & 0,25 & 0,5 & 0,5 & 2 & 2 \\
\hline C2 & $\mathrm{R} 1$ & 3 & 1 & 0,5 & 0,17 & 5 & 0,2 & 0,25 & 2 & 2 & 2 & 2 \\
\hline $\mathrm{C} 3$ & $\mathrm{R} 1$ & 3 & 2 & 1 & 0,17 & 6 & 2 & 0,25 & 2 & 4 & 2 & 2 \\
\hline $\mathrm{C} 4$ & $\mathrm{R} 1$ & 6 & 6 & 6 & 1 & 6 & 6 & 6 & 6 & 6 & 6 & 6 \\
\hline C5 & $\mathrm{R} 1$ & 0,2 & 0,2 & 0,17 & 0,17 & 1 & 0,25 & 0,2 & 0,25 & 0,25 & 0,25 & 0,25 \\
\hline C6 & $\mathrm{R} 1$ & 3 & 5 & 0,5 & 0,17 & 4 & 1 & 0,2 & 2 & 4 & 4 & 3 \\
\hline C7 & $\mathrm{R} 1$ & 4 & 4 & 4 & 0,17 & 5 & 5 & 1 & 3 & 5 & 5 & 3 \\
\hline $\mathrm{C} 8$ & $\mathrm{R} 1$ & 2 & 0,5 & 0,5 & 0,17 & 4 & 0,5 & 0,33 & 1 & 2 & 1 & 2 \\
\hline C9 & $\mathrm{R} 1$ & 2 & 0,5 & 0,25 & 0,17 & 4 & 0,25 & 0,2 & 0,5 & 1 & 1 & 0,5 \\
\hline $\mathrm{C} 10$ & $\mathrm{R} 1$ & 0,5 & 0,5 & 0,5 & 0,17 & 4 & 0,25 & 0,2 & 1 & 1 & 1 & 0,5 \\
\hline $\mathrm{C} 11$ & $\mathrm{R} 1$ & 0,5 & 0,5 & 0,5 & 0,17 & 4 & 0,33 & 0,33 & 0,5 & 2 & 2 & 1 \\
\hline Total & & 25,2 & 20,5 & 14,3 & 2,67 & 48 & 16,1 & 9,22 & 18,8 & 27,8 & 26,3 & 22,3 \\
\hline
\end{tabular}

Table 5.

CR calculation responden 1

\begin{tabular}{lllll}
\hline \hline Criteria & & Priority vector & Total Weight Priority & Consistency measure \\
\hline C1 & R1 & 0,045868 & 0,532497497 & 11,609418 \\
C2 & R1 & 0,068532 & 0,812423279 & 11,8546174 \\
C3 & R1 & 0,094749 & 1,214294451 & 12,8159632 \\
C4 & R1 & 0,319008 & 4,404960808 & 13,8083153 \\
C5 & R1 & 0,017547 & 0,214391977 & 12,2179768 \\
C6 & R1 & 0,105931 & 1,343500044 & 12,6827325 \\
C7 & R1 & 0,171375 & 2,362197122 & 13,7837647 \\
C8 & R1 & 0,05502 & 0,668305045 & 12,1466799 \\
C9 & R1 & 0,03887 & 0,460472774 & 11,8463582 \\
C10 & R1 & 0,037478 & 0,442868129 & 11,8166575 \\
C11 & R1 & 0,045621 & 0,546195385 & 11,9723624 \\
& & & CI & 12,4140769 \\
& & CR & 0,14140769 \\
\hline
\end{tabular}

Table 6.

Fuzzy number criteria matrix

\begin{tabular}{|c|c|c|c|c|c|c|c|c|c|c|c|c|c|c|c|c|c|c|c|c|c|c|c|c|c|c|c|c|c|c|c|c|c|}
\hline \multirow{2}{*}{$\begin{array}{l}\text { Crit } \\
\text { eria }\end{array}$} & \multicolumn{3}{|c|}{$\mathrm{Cl}$} & \multicolumn{3}{|c|}{$\mathrm{C} 2$} & \multicolumn{3}{|c|}{$\mathrm{C} 3$} & \multicolumn{3}{|c|}{$\mathrm{C} 4$} & \multicolumn{3}{|c|}{ C5 } & \multicolumn{3}{|c|}{ C6 } & \multicolumn{3}{|c|}{$\mathrm{C} 7$} & \multicolumn{3}{|c|}{$\mathrm{C} 8$} & \multicolumn{3}{|c|}{$\mathrm{C} 9$} & \multicolumn{3}{|c|}{$\mathrm{C} 10$} & \multicolumn{3}{|c|}{$\mathrm{Cll}$} \\
\hline & 1 & $\mathrm{~m}$ & $\mathrm{u}$ & 1 & $\mathrm{~m}$ & $\mathrm{u}$ & 1 & $\mathrm{~m}$ & $\mathrm{u}$ & 1 & $\mathrm{~m}$ & u & 1 & $\mathrm{~m}$ & $\mathrm{u}$ & 1 & $\mathrm{~m}$ & $\mathrm{u}$ & 1 & $\mathrm{~m}$ & u & 1 & $\mathrm{~m}$ & $\mathrm{u}$ & 1 & $\mathrm{~m}$ & $\mathrm{u}$ & 1 & $\mathrm{~m}$ & u & 1 & $\mathrm{~m}$ & $\mathrm{u}$ \\
\hline $\mathrm{Cl}$ & 1 & 1 & 1 & $\begin{array}{c}0, \\
29\end{array}$ & $\begin{array}{l}0, \\
56\end{array}$ & $\begin{array}{l}1, \\
38\end{array}$ & $\begin{array}{c}0, \\
29\end{array}$ & $\begin{array}{l}0, \\
56\end{array}$ & $\begin{array}{l}1, \\
38\end{array}$ & $\begin{array}{c}0, \\
14\end{array}$ & 0 & 0 , & $\begin{array}{l}0, \\
9\end{array}$ & $\begin{array}{l}1, \\
72\end{array}$ & $\begin{array}{l}2, \\
81\end{array}$ & 0, & 0, & $\begin{array}{l}, \\
8\end{array}$ & $\begin{array}{l}0, \\
38\end{array}$ & $\begin{array}{l}0, \\
82\end{array}$ & $\begin{array}{l}, \\
66\end{array}$ & $\begin{array}{c}0, \\
42\end{array}$ & 0, & $\begin{array}{l}1, \\
9\end{array}$ & 0, & $\begin{array}{l}0, \\
55\end{array}$ & $\begin{array}{l}\text { l, } \\
11\end{array}$ & $\begin{array}{c}0, \\
27\end{array}$ & 0 & $\begin{array}{l}1, \\
06\end{array}$ & $\begin{array}{l}0, \\
55\end{array}$ & $\begin{array}{l}1, \\
25\end{array}$ & $\begin{array}{l}2, \\
51\end{array}$ \\
\hline $\mathrm{C}_{2}$ & $\begin{array}{l}0, \\
72\end{array}$ & $\begin{array}{l}1, \\
78\end{array}$ & $\begin{array}{l}3 \\
47\end{array}$ & 1 & 1 & 1 & 0, & $\begin{array}{l}30 \\
0, \\
57\end{array}$ & $\begin{array}{l}30 \\
2\end{array}$ & $\begin{array}{l}14 \\
0 \\
14\end{array}$ & $\begin{array}{l}1 \\
0, \\
19\end{array}$ & $\begin{array}{l}0, \\
3\end{array}$ & $\begin{array}{l}1 \\
1, \\
72\end{array}$ & $\begin{array}{l}3, \\
63\end{array}$ & $\begin{array}{l}81 \\
5, \\
75\end{array}$ & $\begin{array}{l}0 \\
0, \\
49\end{array}$ & $\begin{array}{l}0, \\
67\end{array}$ & $\begin{array}{l}0 \\
1, \\
06\end{array}$ & $\begin{array}{l}30 \\
0, \\
87\end{array}$ & $\begin{array}{l}2 \\
1, \\
05\end{array}$ & $\begin{array}{l}1, \\
1, \\
28\end{array}$ & 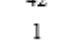 & $\begin{array}{l}74 \\
1, \\
78\end{array}$ & $\begin{array}{l}2 \\
21\end{array}$ & $\begin{array}{l}20 \\
0, \\
72\end{array}$ & 1, & $\begin{array}{l}11 \\
82\end{array}$ & & $\frac{6}{2}$ & $\begin{array}{l}1, \\
32\end{array}$ & 1 & $\begin{array}{l}1 \\
43\end{array}$ & $\begin{array}{l}1, \\
82\end{array}$ \\
\hline C3 & $\begin{array}{l}0, \\
72\end{array}$ & $\begin{array}{l}1 \\
78\end{array}$ & $\begin{array}{l}3 \\
47\end{array}$ & $\begin{array}{l}0, \\
83\end{array}$ & $\begin{array}{l}1 \\
74\end{array}$ & $\begin{array}{l}3 \\
44\end{array}$ & 1 & 1 & 1 & $\begin{array}{l}0, \\
14\end{array}$ & $\begin{array}{c}0 \\
19\end{array}$ & $\begin{array}{l}0, \\
3\end{array}$ & $\begin{array}{l}1, \\
82\end{array}$ & $\begin{array}{l}3 \\
76\end{array}$ & $\begin{array}{l}5, \\
91\end{array}$ & $\begin{array}{l}0 \\
72\end{array}$ & $\begin{array}{l}1 \\
15\end{array}$ & $\begin{array}{l}1 \\
82\end{array}$ & $\begin{array}{l}0, \\
63\end{array}$ & $\begin{array}{l}0, \\
84\end{array}$ & $\begin{array}{l}1, \\
28\end{array}$ & $\frac{1,}{25}$ & $\begin{array}{l}1, \\
58\end{array}$ & $\begin{array}{l}1 \\
95\end{array}$ & $\begin{array}{l}0, \\
92\end{array}$ & 1 & $\begin{array}{l}1, \\
53\end{array}$ & $\begin{array}{l}0, \\
68\end{array}$ & $\begin{array}{l}1 \\
04\end{array}$ & $\begin{array}{l}1 \\
46\end{array}$ & $\begin{array}{l}0, \\
68\end{array}$ & $\begin{array}{l}1 \\
04\end{array}$ & $\begin{array}{l}1, \\
46\end{array}$ \\
\hline $\mathrm{C} 4$ & 3, & $\begin{array}{l}4, \\
86\end{array}$ & $\begin{array}{l}7 \\
04\end{array}$ & $\begin{array}{l}3, \\
35\end{array}$ & $\begin{array}{l}5 \\
19\end{array}$ & $\begin{array}{l}7 \\
4\end{array}$ & $\begin{array}{l}3, \\
35\end{array}$ & $\begin{array}{l}5 \\
19\end{array}$ & $\begin{array}{l}7 \\
4\end{array}$ & 1 & 1 & 1 & $\begin{array}{l}3, \\
02\end{array}$ & $\begin{array}{l}4, \\
86\end{array}$ & $\begin{array}{l}7 \\
04\end{array}$ & $\begin{array}{l}3, \\
02\end{array}$ & $\begin{array}{l}4, \\
86\end{array}$ & $\begin{array}{l}7 \\
04\end{array}$ & $\begin{array}{l}3, \\
02\end{array}$ & $\begin{array}{l}4, \\
86\end{array}$ & $\begin{array}{l}7, \\
04\end{array}$ & $\begin{array}{l}2, \\
83\end{array}$ & $\begin{array}{l}4, \\
62\end{array}$ & $\begin{array}{l}6 \\
76\end{array}$ & $\begin{array}{l}3, \\
02\end{array}$ & $\begin{array}{l}4, \\
86\end{array}$ & 04 & $\begin{array}{l}3, \\
02\end{array}$ & $\begin{array}{l}4, \\
86\end{array}$ & $\begin{array}{l}7, \\
04\end{array}$ & $\begin{array}{l}3, \\
02\end{array}$ & $\begin{array}{l}4, \\
86\end{array}$ & $\begin{array}{l}7, \\
04\end{array}$ \\
\hline C5 & $\begin{array}{c}0, \\
36\end{array}$ & $\begin{array}{l}0 \\
58\end{array}$ & $\begin{array}{l}1 \\
11\end{array}$ & $\begin{array}{l}0, \\
17\end{array}$ & $\begin{array}{l}0, \\
28\end{array}$ & $\begin{array}{l}0, \\
58\end{array}$ & $\begin{array}{l}0, \\
17\end{array}$ & $\begin{array}{l}0, \\
27\end{array}$ & $\begin{array}{l}0, \\
55\end{array}$ & $\begin{array}{l}0 \\
14\end{array}$ & $\begin{array}{l}0 \\
21\end{array}$ & $\begin{array}{l}0 \\
33\end{array}$ & 1 & 1 & 1 & $\begin{array}{c}0, \\
23\end{array}$ & $\begin{array}{l}0, \\
32\end{array}$ & $\begin{array}{c}0, \\
56\end{array}$ & $\begin{array}{l}0, \\
36\end{array}$ & $\begin{array}{l}0 \\
47\end{array}$ & $\begin{array}{l}0 \\
8\end{array}$ & $\begin{array}{c}0, \\
27\end{array}$ & $\begin{array}{l}0, \\
39\end{array}$ & $\begin{array}{l}0, \\
87\end{array}$ & $\begin{array}{l}0, \\
24\end{array}$ & $\begin{array}{c}0, \\
33\end{array}$ & $\begin{array}{l}0, \\
63\end{array}$ & 0 & $\begin{array}{l}0, \\
3\end{array}$ & $\begin{array}{l}0, \\
51\end{array}$ & $\begin{array}{l}0, \\
25\end{array}$ & $\begin{array}{l}0, \\
35\end{array}$ & . \\
\hline $\mathrm{C} 6$ & 1, & $\begin{array}{l}3 \\
06\end{array}$ & $\begin{array}{l}5, \\
11\end{array}$ & $\begin{array}{l}0, \\
94\end{array}$ & $\begin{array}{l}1, \\
5\end{array}$ & $\begin{array}{l}2, \\
04\end{array}$ & $\begin{array}{l}0, \\
55\end{array}$ & $\begin{array}{l}0, \\
87\end{array}$ & $\begin{array}{l}1 \\
38\end{array}$ & $\begin{array}{l}0 \\
14\end{array}$ & $\begin{array}{l}0 \\
21\end{array}$ & $\begin{array}{l}0, \\
33\end{array}$ & $\begin{array}{l}1, \\
78\end{array}$ & $\begin{array}{l}3 \\
13\end{array}$ & $\begin{array}{c}4 \\
3\end{array}$ & 1 & 1 & 1 & $\begin{array}{l}0, \\
84\end{array}$ & $\frac{1}{25}$ & $\begin{array}{l}1 \\
63\end{array}$ & $\begin{array}{l}1, \\
25\end{array}$ & $\begin{array}{l}1, \\
97\end{array}$ & $\begin{array}{l}2, \\
69\end{array}$ & $\begin{array}{l}0, \\
83\end{array}$ & $\begin{array}{l}1, \\
32\end{array}$ & $\begin{array}{l}1, \\
97\end{array}$ & $\begin{array}{l}1, \\
15\end{array}$ & $\begin{array}{l}1, \\
64\end{array}$ & $\begin{array}{l}1, \\
97\end{array}$ & 1 & $\begin{array}{l}1, \\
55\end{array}$ & $\begin{array}{l}\text {, } \\
9\end{array}$ \\
\hline $\mathrm{C} 7$ & $\begin{array}{l}0, \\
53\end{array}$ & $\begin{array}{l}1, \\
22\end{array}$ & $\begin{array}{l}2, \\
61\end{array}$ & $\begin{array}{l}0, \\
78\end{array}$ & $\begin{array}{l}0, \\
96\end{array}$ & $\begin{array}{l}1, \\
15\end{array}$ & $\begin{array}{l}0, \\
78\end{array}$ & $\begin{array}{l}1 \\
19\end{array}$ & $\begin{array}{l}1, \\
58\end{array}$ & $\begin{array}{l}0, \\
14\end{array}$ & $\begin{array}{l}0, \\
21\end{array}$ & $\begin{array}{l}0, \\
33\end{array}$ & $\begin{array}{l}1, \\
25\end{array}$ & $\begin{array}{l}2 \\
14\end{array}$ & $\begin{array}{l}2, \\
81\end{array}$ & $\begin{array}{l}0, \\
61\end{array}$ & $\begin{array}{l}0, \\
8\end{array}$ & $\begin{array}{c}1 \\
18\end{array}$ & . & 1 & 1 & 1 & $\begin{array}{l}2, \\
05\end{array}$ & $\begin{array}{l}3 \\
31\end{array}$ & $\begin{array}{l}0, \\
65\end{array}$ & $\begin{array}{l}1, \\
17\end{array}$ & $\begin{array}{l}2, \\
57\end{array}$ & $\begin{array}{l}0, \\
65\end{array}$ & $\begin{array}{l}1, \\
17\end{array}$ & $\begin{array}{l}2 \\
57\end{array}$ & $\begin{array}{c}0, \\
72\end{array}$ & $\begin{array}{l}1, \\
32\end{array}$ & 4 \\
\hline $\mathrm{CB}$ & $\begin{array}{l}0, \\
53\end{array}$ & $\begin{array}{l}1, \\
06\end{array}$ & $\begin{array}{l}2 \\
4\end{array}$ & $\begin{array}{l}0, \\
4\end{array}$ & $\begin{array}{l}0, \\
56\end{array}$ & 1 & $\begin{array}{l}0, \\
51\end{array}$ & $\begin{array}{l}0, \\
63\end{array}$ & $\begin{array}{l}0, \\
8\end{array}$ & $\begin{array}{l}0, \\
15\end{array}$ & $\begin{array}{c}0, \\
22\end{array}$ & $\begin{array}{l}0, \\
35\end{array}$ & $\begin{array}{c}1 \\
15\end{array}$ & $\begin{array}{l}2 \\
55\end{array}$ & $\begin{array}{c}3 \\
76\end{array}$ & $\begin{array}{l}0, \\
37\end{array}$ & $\begin{array}{l}0, \\
51\end{array}$ & $\begin{array}{l}0, \\
8\end{array}$ & $\begin{array}{l}0, \\
3\end{array}$ & $\begin{array}{l}0 \\
49\end{array}$ & 1 & 1 & 1 & 1 & $\begin{array}{c}0 \\
47\end{array}$ & $\begin{array}{l}0, \\
72\end{array}$ & $\begin{array}{l}1, \\
26\end{array}$ & 0 & $\begin{array}{l}0, \\
62\end{array}$ & $\begin{array}{l}0, \\
96\end{array}$ & $\begin{array}{c}0, \\
53\end{array}$ & $\begin{array}{l}0, \\
85\end{array}$ & $\begin{array}{l}1, \\
74\end{array}$ \\
\hline $\mathrm{CP}$ & $\begin{array}{l}0, \\
9\end{array}$ & $\begin{array}{l}1, \\
82\end{array}$ & $\begin{array}{l}3 \\
55\end{array}$ & $\begin{array}{l}0, \\
55\end{array}$ & $\begin{array}{l}0, \\
87\end{array}$ & $\begin{array}{l}1, \\
38\end{array}$ & $\begin{array}{l}0, \\
65\end{array}$ & $\begin{array}{l}0, \\
81\end{array}$ & $\begin{array}{l}1, \\
08\end{array}$ & $\begin{array}{l}0, \\
14\end{array}$ & $\begin{array}{c}0, \\
21\end{array}$ & $\begin{array}{l}0, \\
33\end{array}$ & $\begin{array}{l}1, \\
58\end{array}$ & $\begin{array}{l}3, \\
02\end{array}$ & $\begin{array}{l}4, \\
23\end{array}$ & $\begin{array}{l}0, \\
51\end{array}$ & $\begin{array}{c}0, \\
76\end{array}$ & $\begin{array}{l}1, \\
2\end{array}$ & $\begin{array}{l}0, \\
39\end{array}$ & $\begin{array}{l}0, \\
85\end{array}$ & $\begin{array}{l}1 \\
53\end{array}$ & 79 & $\begin{array}{l}1, \\
39\end{array}$ & $\begin{array}{l}2, \\
14\end{array}$ & 1 & 1 & 1 & $\begin{array}{l}0, \\
72\end{array}$ & $\begin{array}{l}0, \\
8\end{array}$ & 1 & $\begin{array}{c}0, \\
76\end{array}$ & $\begin{array}{l}1, \\
08\end{array}$ & $\begin{array}{l}1, \\
38\end{array}$ \\
\hline $\mathrm{C} 10$ & $\begin{array}{l}0, \\
94\end{array}$ & $\begin{array}{l}2 \\
14\end{array}$ & $\begin{array}{l}3 \\
71\end{array}$ & $\begin{array}{l}0, \\
76\end{array}$ & $\begin{array}{l}1, \\
08\end{array}$ & $\begin{array}{l}1, \\
38\end{array}$ & $\begin{array}{l}0, \\
68\end{array}$ & $\begin{array}{l}0, \\
96\end{array}$ & $\begin{array}{c}1 \\
48\end{array}$ & $\begin{array}{l}0, \\
14\end{array}$ & $\begin{array}{l}0 \\
21\end{array}$ & $\begin{array}{l}0, \\
33\end{array}$ & $\begin{array}{l}1, \\
97\end{array}$ & $\begin{array}{l}3, \\
35\end{array}$ & $\begin{array}{l}4, \\
52\end{array}$ & 0, & $\begin{array}{l}0 \\
61\end{array}$ & $\begin{array}{l}0 \\
87\end{array}$ & $\begin{array}{l}0, \\
39\end{array}$ & $\begin{array}{l}0, \\
85\end{array}$ & $\begin{array}{l}1, \\
53\end{array}$ & $\begin{array}{l}1, \\
05\end{array}$ & $\begin{array}{l}1, \\
6\end{array}$ & $\begin{array}{l}2 \\
14\end{array}$ & 1 & $\begin{array}{l}1, \\
25\end{array}$ & $\begin{array}{c}1, \\
38\end{array}$ & 1 & 1 & 1 & $\begin{array}{c}0, \\
0, \\
76\end{array}$ & 1, & 30 \\
\hline $\mathrm{C} 11$ & 0 & $\begin{array}{l}0, \\
8\end{array}$ & $\begin{array}{l}1, \\
82\end{array}$ & 0 & $\begin{array}{l}0, \\
7\end{array}$ & 1 & $\begin{array}{l}0, \\
68\end{array}$ & $\begin{array}{l}0, \\
96\end{array}$ & $\begin{array}{l}1 \\
48\end{array}$ & $\begin{array}{c}0, \\
14\end{array}$ & $\begin{array}{c}0, \\
21\end{array}$ & $\begin{array}{l}0, \\
33\end{array}$ & $\begin{array}{l}1 \\
43\end{array}$ & $\begin{array}{l}2 \\
83\end{array}$ & 4, & $\begin{array}{l}0, \\
53\end{array}$ & $\begin{array}{l}0 \\
64\end{array}$ & 1 & $\begin{array}{c}0 \\
42\end{array}$ & $\begin{array}{l}0, \\
76\end{array}$ & $\begin{array}{l}1, \\
38\end{array}$ & $\begin{array}{l}0, \\
57\end{array}$ & $\begin{array}{l}1, \\
18\end{array}$ & 1, & $\begin{array}{l}0, \\
72\end{array}$ & $\begin{array}{l}0, \\
92\end{array}$ & $\begin{array}{l}1, \\
32\end{array}$ & $\begin{array}{l}0, \\
53\end{array}$ & 74 & $\begin{array}{l}1 \\
32\end{array}$ & 1 & 1 & 1 \\
\hline
\end{tabular}

\section{3) Perform the Fuzzy Element Defuzzification Process}

Perform the fuzzy element defuzzification process using the Center of area (COA) method.

$$
M_{i}=\frac{\left(l w_{i}, m w_{i}, u w_{i}\right)}{3}
$$

\section{4) Normalization of Mi Values}

Normalization of Mi values by calculation:

$$
N_{i}=\frac{M_{i}}{\sum_{i=1}^{n} M_{i}}
$$

This normalized priority weight calculation procedure must be applied to the evaluation of specific alternatives for each criterion (alternative preference matrix).

\section{APPLICATION}

A. AHP

\section{1) Criteria Obtained from The Results of The Interview}

Interviews were conducted with experts in companies related to heavy equipment maintenance. These experts are from the maintenance department as the maintenance 
The $6^{\text {th }}$ International Seminar on Science and Technology (ISST) 2020

July $25^{\text {th }} 2020$, Institut Teknologi Sepuluh Nopember, Surabaya, Indonesia

Table 7.

Criteria weighting

\begin{tabular}{lc}
\hline \hline Criteria & Weight \\
\hline Cost & 0,0571 \\
Quality & 0,076912 \\
Availability & 0,085194 \\
Safety & 0,305789 \\
Issued Report & 0,0314 \\
Machine Performance & 0,093847 \\
Government Regulation & 0,081697 \\
House Keeping & 0,055706 \\
Respond Time & 0,071048 \\
Manpower Quality & 0,077363 \\
Maintenance Time & 0,063945 \\
\hline \hline
\end{tabular}

Table 8 .

Alternative score

\begin{tabular}{|c|c|c|c|c|c|c|c|c|c|c|c|c|}
\hline \multirow[b]{2}{*}{ Altemative } & \multicolumn{11}{|l|}{ Score } & \multirow[b]{2}{*}{ Total } \\
\hline & Cost & Quality & Availability & Safety & $\begin{array}{l}\text { Isgued } \\
\text { Report }\end{array}$ & $\begin{array}{l}\text { Machine } \\
\text { Performance }\end{array}$ & $\begin{array}{l}\text { Government } \\
\text { Regulation }\end{array}$ & $\begin{array}{l}\text { House } \\
\text { Keeping }\end{array}$ & $\begin{array}{l}\text { Respond } \\
\text { Time }\end{array}$ & $\begin{array}{l}\text { Manpower } \\
\text { Quality }\end{array}$ & $\begin{array}{l}\text { Maintenance } \\
\text { Time }\end{array}$ & \\
\hline MARC & 0,00823 & 0,03723 & 0,039654612 & 0,0832 & 0,01212 & 0,041753758 & 0,016268298 & 0,02083 & 0,030803 & 0,0299164 & 0,029025891 & 0,349036 \\
\hline SSA & 0,02122 & 0,02196 & 0,027269219 & 0,0804 & 0,0105 & 0,031436101 & 0,016464077 & 0,02103 & 0,022696 & 0,029327 & 0,022151753 & 0,304452 \\
\hline ON CALL & 0,01866 & 0,00838 & 0,00802251 & 0,05146 & 0,00497 & 0,012076001 & 0,012510194 & 0,00819 & 0,00867 & 0,0069226 & 0,006266449 & 0,146123 \\
\hline PT. VALE & 0,00899 & 0,00934 & 0,010247631 & 0,09073 & 0,00382 & 0,008581405 & 0,036454454 & 0,00565 & 0,008879 & 0,0111972 & 0,006500529 & 0,20039 \\
\hline
\end{tabular}

executor and from the SCM department as the part that carries out the agreement / contract with the contractor.

\section{2) Pair-Wise Comparison}

Pair-wise comaparison using questionnaire to 5 respondent. Result of from the questionnaire shown in the example responden 1 Table 4. After find the example responden in Table 4, next step is to find the Consistenncy Ratio. Consistency ratio for responden 1 shown in Table 5.

From the Table 5, CR value $0,09364748<0,100$, so the data is consistent.

\section{B. Fuzzy AHP}

Data from respondents is converted into triangular fuzzy numbers (TFN) using scale in Table 3 mention before. Matrix Fuzzy number matrix of 5 respondents were converted using the geometric mean method, and got the results in Table 6. Following process fuzzy step mention in section II, result of criteria weighting shown in Table 7.

After result in Table 7, process continue to find score of alternative fo each criteria, the result shown in Table 8 .

From the Table 8 shows that the MARC alternative has the greatest value compared to the three other alternatives that is equal to 0.3490 . The second alternative is SSA worth 0.2003 and the alternative with the smallest weight is the on call alternative.

\section{CONCLUSION}

Fuzzy AHP is a method for dealing with uncertainty and helps decision makers in complex problems with various conflicting criteria. Fuzzy AHP is better method to reduce subjective comparing to AHP. Research shows that the method can be used to choose strategy maintenance in company and as the result is MARC alternative is the best strategy of maintenance that company should use with high score in quality aspect.

\section{REFERENCES}

[1] Thomas L. Saaty, Luis G. Vargas., Decision Making With The Analytic Network Process. Springer, 2006.

[2] Sara Cullen., The Contract Scorecard. Successful Outsourcing by Design. Gower Publishing Limited, 2009.

[3] S.M. Ahmed, C.L. Karmaker., Supply Chain Contract Selection Using Delphi-based AHP: A Case Study in the Bangladeshi Super Shop. Journal of Supply Chain Management Systems. 8 (3) 2019, 37-47. http://publishingindia.com/jscms/.

[4] Romulo Luciano., The Hazards of Misusing the Smart Contract: An AHP Approach to Its Risk. Journal of Information Security, 2019, 10, 25-44.

[5] P Z Razi, N I Ramli, M I Ali and P J Ramadhansyah., Selection of Best Consultant by using Analytical Hierarchy Process (AHP). Article in IOP Conference Series Materials Science and Engineering . January 2020, Malaysia.

[6] Agnieszka Lesniak, Daniel Kubek, Edyta Plebankiewicz, Krzysztof Zima and Stanisław Belniak., Fuzzy AHP Application for Supporting Contractors'Bidding Decision. Journal Symmetry 2018, 10, 642; doi:10.3390/sym10110642. Poland, 2018.

[7] Sara Najiazarpour \& Homa Pouresfandyani., Assessment and Selection of Contractors in Specific Contracting Projects with Supply Chain Approach, Using GRAY and AHP Methods as Decision Support. Published by Canadian Center of Science and Education, 2018. 